\title{
Paradoxical Aging Changes of the Atrioventricular Nodal Properties in Patients With Atrioventricular Nodal Re-Entrant Tachycardia
}

\author{
Tzu-Wei Tseng, MD; Yu-Feng Hu, MD; Chin-Feng Tsai, MD; Hsuan-Ming Tsao, MD; \\ Ching-Tai Tai, MD; Yenn-Jiang Lin, MD; Shih-Lin Chang, MD; Li-Wei Lo, MD; \\ Pi-Chang Lee, MD; Cheng-Hung Li, MD; Tze-Fan Chao, MD; Kazuyoshi Suenari, MD; \\ Yung-Kuo Lin, MD; Chern-En Chiang, MD; Shih-Ann Chen, MD
}

\begin{abstract}
Background: This study aimed to investigate the impact of aging on electrophysiological characteristics in patients with atrioventricular nodal re-entrant tachycardia (AVNRT).

Methods and Results: The 2,111 patients who underwent an electrophysiological study and radiofrequency (RF) catheter ablation of AVNRT were enrolled. The patients were divided into 4 groups according to age (group 1: $<20$ years; group 2: 20-39 years; group 3: 40-59 years; and group 4: $\geq 60$ years). The gender distribution differed with age. The atrio-Hisian interval, and effective refractory periods (ERP) of the right atrium, ventricle, antegrade slow pathway, retrograde slow pathway and fast pathway, and tachycardia cycle length all increased with age. However, a paradoxical change in the fast pathway ERP was noted. The fast pathway ERP was significantly longer in group 2 than in other groups, and was associated with the largest tachycardia window. The response to catecholamines was similar between different age groups. Procedure time, radiation time, and complications did not differ. However, the number of RF impulses was higher in group 2 compared with other groups (7.6 \pm 9.3 , $\mathrm{P}=0.04$ ), which might imply a differing complexity during the ablation.
\end{abstract}

Conclusions: Paradoxical aging changes of AVN electrophysiological characteristics were associated with a different atrioventricular nodal conduction property and the number of RF impulses. (Circ J 2011; 75: 15811584)

Key Words: Age; Atrioventricular nodal re-entrant tachycardia; Catheter ablation

A ge-related changes have been reported in different cardiovascular diseases. ${ }^{1,2}$ Although atrioventricular nodal re-entry tachycardia (AVNRT) has been the most common paroxysmal supraventricular tachycardia, few studies have reported age-related changes in electrophysiological properties of the atrioventricular (AV) node in detail. Blaufox et al reported that adolescents had a longer fast pathway effective refractory period (ERP), slow pathway ERP, and atrio-Hisian (AH) interval than children. ${ }^{3}$ Meiltz et al reported that patients who were older than 65 years had a longer mean AH interval, Hisian-ventricle interval, fast pathway ERP, and tachycardia cycle length than the younger patient groups. ${ }^{4}$
Whether the complications after the ablation of AVNRT in older people become higher remains controversial. Most studies reported that recurrences were less common in older people., ${ }^{4,5}$ Those studies were limited by their study design, and the reported electrophysiological parameters remained limited.

An investigation of the differences with aging might lead to a better understanding of the mechanism of the occurrence of AVNRT. Therefore, the present study aimed to compare age-related changes in electrophysiological parameters and results after catheter ablation.

Received December 7, 2010; revised manuscript received January 20, 2011; accepted February 22, 2011; released online April 22, 2011 Time for primary review: 16 days

Division of Cardiology, Taipei Veterans General Hospital, Taipei (T.-W.T., Y.-F.H., C.-T.T., Y.-J.L., S.-L.C., L.-W.L., C.-H.L., T.-F.C., K.S., C.-E.C., S.-A.C.); Department of Medicine and Institute of Clinical Medicine, Cardiovascular Research Center, National YangMing University School of Medicine, Taipei (Y.-F.H., C.-T.T., Y.-J.L., S.-L.C., L.-W.L., P.-C.L., C.-H.L., T.-F.C., C.-E.C., S.-A.C.); Division of Pediatric Cardiology, Taipei Veterans General Hospital, Taipei (P.-C.L.); School of Medicine, Chung Shan Medical University, Taichung (C.-F.T.); Division of Cardiology, National Yang-Ming University Hospital, Yilan (H.-M.T.); and Division of Cardiology, Taipei Wan Fang Hospital, Taipei (Y.-K.L.), Taiwan

The first two authors contributed equally to this study (T.-W.T., Y.-F.H.).

Mailing address: Shih-Ann Chen, MD, Division of Cardiology, Taipei Veterans General Hospital, 201 Sec.2, Shih-Pai Road, Taipei,

Taiwan. E-mail: epsachen@ms41.hinet.net

ISSN-1346-9843 doi:10.1253/circj.CJ-10-1205

All rights are reserved to the Japanese Circulation Society. For permissions, please e-mail: cj@j-circ.or.jp 


\section{Editorial p 1567}

\section{Methods}

\section{Study Population and Electrophysiological Study}

A total of 2,111 consecutive AVNRT patients who were referred to Taipei Veterans General Hospital for an electrophysiological study (EPS) and radiofrequency (RF) catheter ablation were enrolled in the study. The present study only enrolled AVNRT patients who received the primary procedure and excluded patients who received secondary procedures. The patients were divided into 4 groups according to age (group 1: <20 years; group 2: 20-39 years; group 3: 4059 years; and group $4 \geq 60$ years).

As described previously, each patient underwent a baseline EPS in the fasting, non-sedated state, and all antiarrhythmic drugs were discontinued for a minimum of 5 drug half-lives before the procedure. ${ }^{6,7}$ A $7 \mathrm{~F}$ decapolar catheter with a $2 \mathrm{~mm}$ interelectrode distance and $5 \mathrm{~mm}$ spacing between each electrode pair was placed in the coronary sinus. The position of the proximal electrode pair at the ostium of the coronary sinus was confirmed with a contrast injection. Three multipolar, deflectable-tip, closely spaced (interelectrode spacing, $2 \mathrm{~mm}$ ) electrode catheters were placed in the high right atrium, Hisbundle area, and right ventricle. At the beginning of the study, programmed electrical stimulation consisting of atrial and ventricular incremental pacing and extrastimulation in $10-\mathrm{ms}$ decrements was performed to ascertain the anterograde and retrograde AV nodal conduction properties and inducibility of AVNRT. Intravenous isoproterenol (at graded dosages from 1 to $4 \mu \mathrm{g} / \mathrm{min}$ ) was used to facilitate induction of the tachycardia after attempting to induce it with pacing maneuvers. If a tachycardia was not inducible under isoproterenol infusion, intravenous atropine $(0.5-1 \mathrm{mg})$ was used.

\section{Definitions}

Details of the diagnostic EPS and diagnostic criteria of AVNRT have been well described previously. ${ }^{8-12}$ In brief, dual AV nodal pathway curves were characterized by a jump $(\geq 50 \mathrm{~ms})$ in the $\mathrm{H} 1-\mathrm{H} 2$ interval at a critical range of $\mathrm{A} 1-\mathrm{A} 2$ coupling intervals $(10 \mathrm{~ms}$ decrements), resulting in a discontinuity between the portion of the curve to the right of the jump in the $\mathrm{H} 1-\mathrm{H} 2$ interval (fast pathway conduction) and the portion of the curve to the left of the jump (slow pathway conduction). A multiple anterograde AV nodal pathway physiology was defined by AV nodal conduction curves with $\geq 2$ discontinuities. A retrograde fast pathway was defined if the earliest atrial activation of a retrograde pathway was recorded at the His bundle region and revealed no decremental conduction with an almost fixed HA interval during ventricular pacing and ventricular extrastimulus testing. The retrograde slow pathway was defined as the retrograde conduction that occurred after a sudden increment in the ventriculoatrial conduction time of $>50 \mathrm{~ms}$, with a shift in the earliest retrograde atrial activation from the His bundle region to the ostium of the coronary sinus in response to changes in the cycle length or coupling interval; the slow pathway also exhibited decremental conduction. Typical AVNRT was defined as a tachycardia using an anterograde slow pathway and retrograde fast pathway. The other types of AVNRT were defined as atypical AVNRT. If patients had anterograde dual/multiple AV nodal pathways, the difference between the antegrade fast and shortest slow pathway ERP was defined as the tachycardia window. ${ }^{10,13}$

\section{Mapping and Ablation Procedure}

The method used for mapping and ablation has been previously described. ${ }^{6,7}$ To determine the possible anatomic site of the slow pathway, the mapping and ablation catheter tip was initially positioned in the posterior region, then the medial, and finally the anterior region, if necessary. The presumed ablation site was considered optimal if the bipolar electrograms obtained from the distal electrode exhibited an atrial/ ventricular electrogram amplitude ratio of 0.1 to 0.5 , with a multicomponent or a putative slow pathway potential. Catheter ablation was performed $\left(40-50 \mathrm{~W}, 50-55^{\circ} \mathrm{C}, 20-60 \mathrm{~s}\right)$ using a 4-mm tip non-irrigated ablation catheter. RF energy was delivered from a generator (EPT-1000, Boston Scientific Co, Natick, MA, USA), and if junctional rhythm did not appear during the first $10 \mathrm{~s}$, the energy delivery was terminated and another ablation site was selected. The energy delivery was also terminated immediately in the event of an increase in the impedance, dislocation of the catheter, prolongation of the PR interval, or occurrence of AV block. An attempt to induce AVNRT with evaluation of the AV nodal conduction properties was conducted immediately after each application of the RF energy. The endpoint of the procedure was the elimination of the anterograde slow pathways with the non-inducibility of AVNRT or a modification of those pathways with only one residual echo beat under an intravenous infusion of isoproterenol. Complications of the AV node were defined as first degree, second degree, and third degree AV nodal block.

\section{Statistical Analysis}

The continuous variables are expressed as mean \pm SD. Comparisons between continuous variables were performed using a one-way analysis of variance (ANOVA), and the nominal variables were compared by a chi-square test for linear trends. A P-value of $<0.05$ was considered statistically significant. All statistical analyses were performed using commercial statistical SPSS version 17.0 software (SPSS, Chicago, IL, USA).

\section{Results}

\section{Basic and Electrophysiological Characteristics (Table 1)}

We enrolled 2,111 patients with AVNRT who underwent an electrophysiological procedure in Taipei Veterans General Hospital. The mean age was $48.8 \pm 17.6$ years (11-92 years) and there were 882 males $(41.8 \%)$. The average age of onset of the AVNRT was $39.4 \pm 17.9$ years, and that increased with the different increasing age groups. The gender distribution differed in the 4 age groups. The AH interval, right atrial ERP, right ventricular ERP, antegrade slow pathway, retrograde slow pathway, retrograde fast pathway, and tachycardia cycle length all increased with aging. However, a paradoxical change in the fast pathway ERP was noted. In group 2, the fast pathway ERP was significantly longer than that in the other groups, and was associated with a different antegrade tachycardia window. The incidence of atypical AVNRT was significantly higher in group 2 . The response to catecholamines was similar between the different age groups.

\section{Results of Catheter Ablation (Table 2)}

The mean procedural time was $49.9 \pm 34.9$ min and mean radiation time was $14.0 \pm 12.7 \mathrm{~min}$. The procedure time, radiation exposure time, and complications representing AV nodal insufficiency did not differ. However, the number of RF applications was higher in group 2 compared with the other groups (7.6 \pm 9.3 impulses, $\mathrm{P}=0.041$ ), which might imply a different 


\begin{tabular}{|lccccc|}
\hline \multicolumn{7}{c}{ Table 1. Baseline and Electrophysiological Characteristics } & & & & \\
\multicolumn{1}{c}{ Age group (years) } & $<\mathbf{2 0}$ & $\mathbf{2 0 - 3 9}$ & $\mathbf{4 0 - 5 9}$ & $\mathbf{6 0}$ & P value \\
Patient number & 103 & 558 & 786 & 664 & \\
Female & $57(55.3 \%)$ & $367(65.8 \%)$ & $549(69.8 \%)$ & $261(39.3 \%)$ & $<0.001$ \\
Onset age (years) & $12.8 \pm 3.0$ & $21.5 \pm 7.5$ & $38.5 \pm 11.4$ & $56 \pm 12.4$ & $<0.001$ \\
Atrial ERP (ms) & $192.2 \pm 31.9$ & $202.5 \pm 34.9$ & $210.5 \pm 32.4$ & $221.2 \pm 35.1$ & $<0.01$ \\
Atrio-Hisian interval (ms) & $80.0 \pm 38.8$ & $78.0 \pm 22.0$ & $78.3 \pm 25.2$ & $86.9 \pm 28.8$ & $<0.01$ \\
Ventricular ERP (ms) & $211.4 \pm 21.1$ & $216.2 \pm 24.6$ & $220.6 \pm 23.3$ & $223.9 \pm 25.3$ & $<0.01$ \\
Antegrade fast ERP (ms) & $312.2 \pm 74.8$ & $331.0 \pm 79.7$ & $320.2 \pm 68.6$ & $324.4 \pm 67.1$ & 0.02 \\
Antegrade slow ERP (ms) & $263.3 \pm 38.6$ & $278.4 \pm 54.7$ & $275.9 \pm 44.1$ & $289.8 \pm 49.9$ & $<0.01$ \\
Retrograde fast ERP (ms) & $283.4 \pm 81.3$ & $293.8 \pm 81.8$ & $293.5 \pm 119.2$ & $308.5 \pm 86.1$ & 0.02 \\
Retrograde slow ERP (ms) & $257.6 \pm 74.6$ & $292.4 \pm 68.0$ & $298.5 \pm 66.3$ & $318.0 \pm 75.4$ & 0.002 \\
Tachycardia cycle length (ms) & $336.3 \pm 69.7$ & $345.9 \pm 71.9$ & $345.7 \pm 62.8$ & $359.4 \pm 67.1$ & $<0.01$ \\
Tachycardia window (ms) & $68.3 \pm 60.3$ & $68.8 \pm 57.7$ & $57.9 \pm 50.2$ & $55.9 \pm 45.5$ & $<0.01$ \\
Catecholamine sensitive (\%) & 41.3 & 38.6 & 40.9 & 39.2 & 0.82 \\
Atypical AVNRT & $10(9.7 \%)$ & $86(15.4 \%)$ & $70(8.9 \%)$ & $54(8.1 \%)$ & $<0.01$ \\
\hline
\end{tabular}

ERP, effective refractory period; AVNRT, atrioventricular nodal re-entrant tachycardia.

\begin{tabular}{|lccccc|}
\hline Table 2. Procedure-Related Data and Complications & & & & \\
Age group (years) & $<\mathbf{2 0}$ & $\mathbf{2 0 - 3 9}$ & $\mathbf{4 0 - 5 9}$ & $\mathbf{6 0}$ & P value \\
Procedure time (min) & $45.6 \pm 33.9$ & $49.2 \pm 32.9$ & $49.0 \pm 34.9$ & $52.5 \pm 36.8$ & 0.38 \\
Radiation time (min) & $12.4 \pm 9.0$ & $13.7 \pm 10.7$ & $13.5 \pm 11.6$ & $14.0 \pm 12.7$ & 0.68 \\
RF impulses $(\mathrm{n})$ & $6.2 \pm 6.9$ & $7.6 \pm 9.3$ & $6.3 \pm 8.5$ & $6.2 \pm 9.0$ & 0.04 \\
AV nodal complication & 0 & $1(0.18 \%)$ & $5(0.64 \%)$ & $7(1.05 \%)$ & 0.39 \\
Secondary procedure & $1(0.9 \%)$ & $9(1.6 \%)$ & $3(0.4 \%)$ & $10(1.5 \%)$ & 0.10 \\
Acute success rate (\%) & 99.0 & 98.4 & 99.6 & 98.5 & 0.98 \\
\hline
\end{tabular}

$\mathrm{RF}$, radiofrequency; $\mathrm{AV}$, atrioventricular.

complexity during the ablation. The incidence of a second procedure did not differ between the different age groups.

\section{Discussion}

\section{Major Findings}

Age-related prolongation of the retrograde slow and fast pathways was noted. Interestingly, a paradoxical change in the fast pathway ERP in patients aged between 20 and 40 years was noted and was associated with a longer tachycardia window and more RF applications required to achieve a successful ablation.

\section{Paradoxical Changes in AV Nodal Electrophysiological Characteristics}

The AV node function has never been investigated in a systemic way, and previous papers were limited by their study design. In a small cohort study it was reported that adolescents had a longer fast pathway ERP, slow pathway ERP, and AH interval than children. ${ }^{3}$ The patients who were older than 75 years had a longer mean $\mathrm{AH}$ interval, Hisian-ventricle interval, fast pathway ERP, and tachycardia cycle length than younger patients. ${ }^{14}$ The present study showed similar findings for the $\mathrm{AH}$ interval, tachycardia cycle length, and slow pathway ERP in a larger cohort, and further provided the agerelated change in retrograde AVN function. The retrograde fast and slow pathway ERP were prolonged with aging. Interestingly, a paradoxical change in the fast pathway ERP in patients aged between 20 and 40 years was noted, which has never been previously reported. Although the mechanism was unclear, the fact that there is a longer antegrade fast pathway ERP in patients aged 20-40 years might imply that there is a different AV nodal remodeling process in young patients with AVNRT. Our previous study showed that the fast antegrade fast ERP in patients with accessory pathways increased with age, and no paradoxical phenomenon was noted. ${ }^{15}$ The wide tachycardia window implied a higher tendency for AVNRT attacks, ${ }^{16}$ and increasing incidence of AVNRT in young patients, which is consistent with previous reports and clinical observations.

Several reports investigated age-related differences.,17,18 Patients aged greater than 65 years had longer antegrade fast AVNERP, which was associated with a longer tachycardia window. An increase in the length of inferior AVN extensions with fibrotic changes in the transitional zone might contribute to a longer AVNERP, dual, or multiple AVN conduction patterns, and the development of AVN re-entry. ${ }^{18}$ The morphology of the AV node changes with aging, and becomes larger with a right inferior extension from 1 year of age until $20 .{ }^{19}$ Age-related fibrofatty tissue could cause the transitional cells to separate and result in changes in the sequence of fast and slow inputs. Our previous report showed that the length between the distal Hisian site and coronary sinus ostium was negatively correlated with age. ${ }^{20}$ The anatomical remodeling with aging might be related to the functional change in the AV node. It remains unclear if patients aged $20-40$ years old in the present study shared similar mechanisms of AVN remodeling as previously reported. How those anatomical and functional aging changes interact still needs further investigation. Three-dimensional electroanatomic mapping has been used to study the functional properties of AVN and different arrhythmias, which might help to study functional and morphological change of AVN with aging in the future. ${ }^{21,22}$

A higher number of RF applications were needed to achieve 
a successful ablation in patients aged 20-40 years in the present study. Differences in the functional properties might be associated with remodeling of the Koch triangle, which led to failure of RF applications in the initial several shots, by traditional anatomical mapping. Therefore, the application number might increase. It remains unclear if different fast AVNERP and the tachycardia window would be associated with a different outcome after catheter ablation. Both Meiltz et al and Haghjoo et al reported that acute success rate, procedure time, and number of RF applications were similar.4,17 However, those studies investigated age-related differences by the cut-off point of 65 years, which might not be applicable to the patients aged 20-40 years in the present study.

\section{Results After Catheter Ablation}

Whether complications increase after the ablation of AVNRT in older people remains controversial. Boulos et al reported that the incidence of complete heart block in the group of patients between 45 and 65 years of age was $2.0 \%$ ( 2 of 102 patients) and for those $>65$ years of age was $8 \%$ (4 of 52 patients). ${ }^{23}$ No patients aged less than 45 years had complete heart block complicating the procedure ( 0 of 117 patients). The age-related differences were highly significant. In contrast, RF ablation of AVNRT was considered highly effective and safe in older people despite a higher prevalence of structural heart disease and longer A-H intervals at baseline., ${ }^{4,14}$ In the present study, the acute success rate, incidence of a second procedure, and complication rate was similar in the different age groups. These findings suggest that catheter ablation is safe and effective regardless of age in a large cohort.

\section{Study Limitations}

This was a single-center retrospective study from a tertiary referral center, which could in part be a referral bias. It remains unclear why the fast AVNERP was longer in the 2040-year-old group, and further mechanistic study might be needed to elucidate the possible reason. Chiu et al reported the outome after catheter ablation of supraventricular tachycardia was favorable in infants and toddlers. ${ }^{24}$ The present study did not enrol those patients.

\section{Conclusions}

The paradoxical aging changes of the AVN electrophysiological characteristics were associated with a different AV nodal conduction property and the number of RF impulses.

\section{Sources of Funding}

This work was supported by research grants from the Taipei Veterans General Hospital (V96C1-049, V97C1-059, V97A-097, V96A-127, V98C1037, V99C1-120), National Scientific Council (NSC95-2314-B-010-017, NSC95-2314-B-010-025, NSC96-2314-B-010-006, NSC96-2628-B-010036, NSC97-2314-B-010-037-MY3, NSC97-2314-B-010-038, NSC982314-B-010-031-MY3).

\section{Disclosure}

Conflict of interest: none declared.

\section{References}

1. Lee HY, Oh BH. Aging and arterial stiffness. Circ $J$ 2010; 74: $2257-2262$.

2. Jugdutt BI. Aging and heart failure: Changing demographics and implications for therapy in the elderly. Heart Fail Rev 2010; 15: 401-405.

3. Blaufox AD, Rhodes JF, Fishberger SB. Age related changes in dual AV nodal physiology. Pacing Clin Electrophysiol 2000; 23:
477-480.

4. Meiltz A, Zimmermann M. Atrioventricular nodal reentrant tachycardia in the elderly: Efficacy and safety of radiofrequency catheter ablation. Pacing Clin Electrophysiol 2007; 30: S103-S107.

5. Zado ES, Callans DJ, Gottlieb CD, Kutalek SP, Wilbur SL, Samuels FL, et al. Efficacy and safety of catheter ablation in octogenarians. $J$ Am Coll Cardiol 2000; 35: 458-462.

6. Chen SA, Wu TJ, Chiang CE, Tai CT, Chiou CW, Ueng KC, et al. Recurrent tachycardia after selective ablation of slow pathway in patients with atrioventricular nodal reentrant tachycardia. Am J Cardiol 1995; 76: $131-137$.

7. Tai CT, Chen SA, Chiang CE, Lee SH, Wen ZC, Chiou CW, et al. Complex electrophysiological characteristics in atrioventricular nodal reentrant tachycardia with continuous atrioventricular node function curves. Circulation 1997; 95: 2541-2547.

8. Lee PC, Hwang B, Tai CT, Hsieh MH, Chen YJ, Chiang CE, et al. The electrophysiological characteristics in patients with ventricular stimulation inducible fast-slow form atrioventricular nodal reentrant tachycardia. Pacing Clin Electrophysiol 2006; 29: 1105-1111.

9. Chen SA, Chiang CE, Tsang WP, Hsia CP, Wang DC, Yeh HI, et al. Selective radiofrequency catheter ablation of fast and slow pathways in 100 patients with atrioventricular nodal reentrant tachycardia. Am Heart J 1993; 125: 1-10.

10. Suenari K, Hu YF, Tsao HM, Tai CT, Chiang CE, Lin YJ, et al. Gender differences in the clinical characteristics and atrioventricular nodal conduction properties in patients with atrioventricular nodal reentrant tachycardia. J Cardiovasc Electrophysiol 2010; 21: $1114-1119$

11. Tai CT, Chen SA, Chiang CE, Cheng CC, Chiou CW, Lee SH, et al. Electrophysiologic characteristics and radiofrequency catheter ablation in patients with multiple atrioventricular nodal reentry tachycardias. Am J Cardiol 1996; 77: 52-58.

12. Tai CT, Chen SA, Chiang CE, Lee SH, Chiou CW, Ueng KC, et al. Multiple anterograde atrioventricular node pathways in patients with atrioventricular node reentrant tachycardia. J Am Coll Cardiol 1996; 28: 725-731.

13. Wimmer AP, Shapiro ML. The window of slow pathway conduction after ablation and recurrence of atrioventricular nodal reentrant tachycardia. J Interv Card Electrophysiol 2002; 6: 51-57.

14. Postock T, Risius T, Ventura R, Klemm HU, Weiss C, Keitel A, et al. Efficacy and safety of radiofrequency catheter ablation of atrioventricular nodal reentrant tachycardia in the elderly. J Cardiovasc Electrophysiol 2005; 16: 608-610.

15. Li CH, Hu YF, Lin YJ, Chang SL, Lo LW, Ta-Chuan T, et al. The impact of age on the electrophysiological characteristics and different arrhythmia patterns in patients with wolf-Parkinson-white syndrome. J Cardiovasc Electrophysiol 2011; 22: 274-279.

16. Liuba I, Jönsson A, Säfström K, Walfridsson H. Gender-related differences in patients with atrioventricular nodal reentry tachycardia. Am J Cardiol 2006; 97: 384-388.

17. Haghjoo M, Arya A, Heidari A, Fazelifar AF, Sadr-Ameli MA. Electrophysiologic characteristics and results of radiofrequency catheter ablation in elderly patients with atrioventricular nodal reentrant tachycardia. J Electrocardiol 2007; 40: 208-213.

18. Grecu M, Floria M, Georgescu CA. Abnormal atrioventricular node conduction and atrioventricular nodal reentrant tachycardia in patients older versus younger than 65 years of age. Pacing Clin Electrophysiol 2009; 32: $98-100$.

19. Kenji W, Kim JS, Becker AE. Morphology of the human atrioventricular node is age dependent: A feature of potential clinical significance. J Cardiovasc Electrophysiol 2000; 11: 1144-1151.

20. Ueng KC, Chen SA, Chiang CE, Tai CT, Lee SH, Chiou CW, et al. Dimension and related anatomical distance of Koch's triangle in patients with atrioventricular nodal reentrant tachycardia. J Cardiovasc Electrophysiol 1996; 7: 1017-1023.

21. Sumitomo N, Tateno S, Nakamura Y, Ushinohama H, Taniguchi K, Ichikawa R. Clinical importance of Koch's triangle size in children: A study using 3-dimensional electroanatomical mapping. Circ J 2007; 71: 1918-1921.

22. Lo LW, Chen SA. Three-dimensional electroanatomic mapping systems in catheter ablation of atrial fibrillation. Circ J 2010; 74: $18-23$.

23. Boulos M, Hoch D, Schecter S, Greenberg S, Levine J. Age dependence of complete heart block complicating radiofrequency ablation of the atrioventricular nodal slow pathway. Am J Cardiol 1998; 82: $390-391$.

24. Chiu SN, Lu CW, Chang CW, Chang CC, Lin MT, Lin JL, et al. Radiofrequency catheter ablation of supraventricular tachycardia in infants and toddlers. Circ J 2009; 73: 1717-1721. 